\title{
Impact of Strain Elastography on BI-RADS classification in small invasive lobular carcinoma
}

\author{
Angelica Rita Chiorean",2, Mădălina Brîndușa Szep', Diana Sorina Feier², Magdalena Duma², \\ Marco Andrei Chiorean ${ }^{3}$, Ștefan Strilciuc ${ }^{4}$
}

${ }^{1}$ Medimages Breast Center, ${ }^{2}$ Department of Radiology, "Iuliu Haţieganu” University of Medicine and Pharmacy, ${ }^{3}$ Department of Surgery, "Ion Chiricuță” Institute of Oncology, ${ }^{4}$ Department of Public Health, Babeș-Bolyai University, Cluj-Napoca, Romania

\begin{abstract}
Aims: The purpose of this study was to determine the impact of strain elastography (SE) on the Breast Imaging Reporting Data System (BI-RADS) classification depending on invasive lobular carcinoma (ILC) lesion size. Materials and methods: We performed a retrospective analysis on a sample of 152 female subjects examined between January 2010 - January 2017. SE was performed on all patients and ILC was subsequently diagnosed by surgical or ultrasound-guided biopsy. BI-RADS $1,2,6$ and Tsukuba BGR cases were omitted. BI-RADS scores were recorded before and after the use of SE. The differences between scores were compared to the ILC tumor size using nonparametric tests and logistic binary regression. We controlled for age, focality, clinical assessment, heredo-collateral antecedents, B-mode and Doppler ultrasound examination. An ROC curve was used to identify the optimal cut-off point for size in relationship to BI-RADS classification difference using Youden's index. Results: The histological subtypes of ILC lesions $(\mathrm{n}=180)$ included in the sample were luminal A $(70 \%, \mathrm{n}=126)$, luminal B $(27.78 \%, \mathrm{n}=50)$, triple negative $(1.67 \%, n=3)$ and HER2 $+(0.56 \%, n=1)$. The BI-RADS classification was higher when SE was performed $(Z=-$ $6.629, \mathrm{p}<0.000)$. The ROC curve identified a cut-off point of $13 \mathrm{~mm}$ for size in relationship to BI-RADS classification difference $(\mathrm{J}=0.670, \mathrm{p}<0.000)$. Small ILC tumors were $17.92 \%$ more likely to influence BI-RADS classification $(\mathrm{p}<0.000)$. Conclusions: SE offers enhanced BI-RADS classification in small ILC tumors $(<13 \mathrm{~mm})$. Sonoelastography brings added value to B-mode breast ultrasound as an adjacent to mammography in breast cancer screening.
\end{abstract}

Keywords: breast; ultrasonography; sonoelastography; invasive lobular carcinoma

\section{Introduction}

According to the American Breast Cancer Society, mortality rates linked to the breast cancer in the United States have dropped between 1989-2015 and are currently stagnating for women aged below 50 years [1]. The World Health Organization (WHO) attributes this to early detection through screening and awareness efforts, as well as breakthroughs in treatment [2]. Nevertheless,

Received 01.10.2017 Accepted 17.01.2018

Med Ultrason

2018, Vol. 20, No 2, 148-153

Corresponding author: Ștefan Strilciuc

7, Pandurilor Street, Universitas Building, 9th Floor

Cluj-Napoca, Romania, 400095

Phone: +40740066761

E-mail: stefan.strilciuc@publichealth.ro breast cancer is one of the leading causes of mortality in women, second only to lung cancer. Surveillance data from the American Cancer Society predicts that 252,710 new cases of invasive and 63,410 of non-invasive (in situ) breast cancer are expected to be diagnosed across the US in 2017. About 40,610 women are expected to die as a result. Many new cases from low- and middleincome countries are diagnosed in late stages [3]. In Eastern Europe, invasive lobular carcinoma (ILC) is the second most prevalent type of breast cancer after invasive ductal carcinoma (IDC) [4]. While prevention remains an important tool for risk reduction in developed countries, the WHO recognizes population-based screening as the cornerstone of cancer control in limited resource settings with weak health systems. Mammography screening is associated with reduction in breast cancer death rates, being the most cost-effective screening tool $[5,6]$. 
The Breast Imaging Reporting and Data System (BIRADS) is a framework created by the American College of Radiology (ACR) to standardize the terminology used in mammographic, magnetic resonance imaging (MRI), and breast ultrasound (US) reports [7]. BI-RADS subdivisions allow quantification of malignancy odds in lesions, increasing the quality of risk assessment in breast cancer $[8,9]$. Breast US is widely used as an adjunct to mammography in screening [10]. In high-risk women, the diagnostic accuracy of US and mammography combined is superior to mammography alone [11]. ILC are sometimes difficult to identify or can be mammographically occult [12]. Small ILC lesions may have subtle US appearance (for example mimicking diffuse mastopathy) and are difficult to visualize. The sensitivity of ultrasound in ILC detection varies between $68-98 \%$ in different studies [13-15].

Elastography is an imaging technique that allows quantification of tissue stiffness. US elastography imaging (EI) individuates benign and malignant breast lesions with high sensitivity and specificity [16-20]. A 2015 study concluded that shear-wave elastography (SWE) can diagnose lobular cancers that have benign findings on conventional imaging [21]. Strain elastography (SE) scores are associated with breast tumor grades [22]. Nevertheless, to our knowledge there are limited studies that explore the added value of SE in small ILC lesions [23].

Correct classification of lesions into BI-RADS categories is essential for predicting the presence of malignancy $[8,24]$. Therefore, the purpose of this study was to determine whether SE has differential impact on BIRADS classification depending on ILC lesion sizes.

\section{Materials and methods}

\section{Study procedures}

This cross-sectional retrospective study was conducted based on international ethical principles for medical research involving human subjects and approved by our Institutional Review Board. Between January 2010 and January 2017, reports of patients referred for screening or diagnosis US were reviewed. Only patients with biopsy confirmed ILC diagnosis and SE examinations were included in the study. Cases with associated IDC or Ductal Carcinoma in Situ were excluded. Our analysis controlled for BI-RADS categories 1, 2, and blue-greenred (BGR) on Tsukuba elasticity score, as well as patients undergoing neoadjuvant therapy (BI-RADS 6).

All examinations were performed by a radiologist with 10 years of experience in SE on a HI VISION Ascendus (Hitachi Ltd., Tokyo, Japan) machine with a Wide Band (6.5-13 MHz) linear probe and an EUB-6500 HiVision (Hitachi Ltd., Tokyo, Japan) machine with linear probe
(36 mm; 5.0-10.0 MHz). The elastogram was obtained in the supine position; a slight, rhythmic compression-decompression movement was applied to the area of interest, aligning the scan plane to the skin surface, anterior margin of the lesion and the chest wall. Techniques were displayed side by side during a real-time elastosonographic examination and classified according to the Ueno-Itoh adaptation of the Tsukuba elasticity score [25,26], taking into account ACR Appropriateness Criteria [27]. Good quality SE images were acquired by applying minimal transducer pressure, to avoid distorting glandular tissue signal. Lesion size was measured in B-mode to avoid differences attributable to elasticity imaging [28]. A 14-gauge core needle and Magnum Bard device (Bard, Inc, NJ, USA) was used to harvest image-guided biopsies. Histologic diagnosis was then extracted from pathologic reports.

B-mode and SE images were reviewed separately for each lesion and 2 BI-RADS scores were recorded for Bmode and SE enhanced examinations. The patients were classified according to the fifth edition of the BI-RADS lexicon, which includes elastographic findings. The system classifies lesions as soft, intermediate, or hard. SE was used to increase BI-RADS scores, taking into account lesion stiffness [29]. Only lesions that appeared hard on SE (4 or 5 elasticity score) were classified in a higher BI-RADS category. Lesions with lower elasticity scores were classified as soft (1 or 2 Tsukuba score) or intermediate ( 3 Tsukuba score).

We defined focality as the region in which breast cancer was located: unifocal - one tumor; multifocal - two or more separate invasive tumors in the same quadrant of the breast; multicentric - two or more separate invasive tumors that occupy more than one quadrant of the same breast [30].

\section{Statistical Analysis}

Data was analyzed by a single operator using SPSS V.23 (IBM, NY, USA). The main variables included in the analysis were lesion size and BI-RADS classification (with and without SE). A per lesion analysis was conducted taking into account multiple lesions per patient as a grouping variable controlled in the regression model. We calculated descriptive statistics for all study variables: number, frequency, mean and standard deviation (SD). We numerically recoded BI-RADS variables and used a Wilcoxon signed rank test to establish a statistically significant difference between BI-RADS classification before and after SE examination. The Wilcoxon signed-rank test is the nonparametric test equivalent to the dependent t-test that does not assume normality in the data. Frequencies of the numeric difference in BI-RADS scores between examinations were interpreted to identify the quantitative impact of SE on the 
BI-RADS scores in our sample. Subsequently, a binary variable was computed depending on the presence of statistically significant differences in BI-RADS scores (values 0 and 1, accordingly). The relationship between size (continuous, independent variable) and BI-RADS difference (binary, dependent variable) was tested using binary logistic regression. Afterwards, an ROC curve (nonparametric assumption, $\mathrm{CI}=95 \%$ ) using lesion size (continuous) as test variable and BI-RADS difference (binary) as state variable was used to calculate the optimal cut-off point. Sensitivity and specificity were weighed equally in the decision process. After coordinating the points of the curve, Youden's J statistic was calculated and compared for each entry, resulting in a clear threshold value for lesion size. To control for possible confounders, the analysis was stratified according to variables collected from the study sample: age, focality, clinical assessment, heredo-collateral antecedents (HCA - self-reported first-degree female relatives diagnosed with breast cancer), B-mode and Doppler US examination. We set the level of significance to $p<0.05$, or confidence interval at $95 \%$.

\section{Results}

The study group comprised 152 females with a total of 180 malignant lesions (biopsy-confirmed ILC) between ages 33-82 (mean \pm SD, 56.6 \pm 10.8$)$. The histological subtypes of ILC were luminal A $(\mathrm{n}=126)$, luminal B $(\mathrm{n}=50)$, triple negative $(\mathrm{n}=3)$ and HER $2+(\mathrm{n}=1)$. Tsukuba elasticity scores ranged from 1 to 5 (mean $\pm \mathrm{SD}, 4.8 \pm 0.6$ ). Tumors included in the analysis measured between 2-75 $\mathrm{mm}$ (mean $\pm \mathrm{SD}, 22.5 \pm 15.1$ ). Values pertaining to SE, Bmode and Doppler examination, focality and clinical assessment are described in Table I.

SE improved BI-RADS classification in $54(30.00 \%)$ of tumors (Table II). The Wilcoxon signed rank test established a statistically significant difference $(Z=-6.629$, $\mathrm{p}<0.000$ ) between BI-RADS classifications before and after SE evaluation (fig 1, fig 2). Out of the entire sample included in the analysis $(n=180)$, SE increased BI-

Table I. BI-RADS classification before and after strain elastography of 180 invasive lobular carcinoma tumors.

\begin{tabular}{lll}
\hline $\begin{array}{l}\text { BI-RADS clas- } \\
\text { sification }\end{array}$ & $\begin{array}{l}\text { Before SE } \\
\mathbf{n = 1 8 0}\end{array}$ & $\begin{array}{l}\text { After SE } \\
\mathbf{n = 1 8 0}\end{array}$ \\
\hline 3 & $2(1.11)$ & $0(0)$ \\
$4 \mathrm{~A}$ & $3(1.66)$ & $2(1.11)$ \\
$4 \mathrm{~B}$ & $24(13.33)$ & $4(2.22)$ \\
$4 \mathrm{C}$ & $30(16.66)$ & $27(15.00)$ \\
5 & $121(67.22)$ & $147(81.66)$ \\
\hline
\end{tabular}

The data are expressed as number (\%); SE - strain elastography; $\mathrm{n}$ - number of malignant lesions
RADS classification in 54 tumors (mean $\pm \mathrm{SD}, 1.23 \pm 0.35$, range $=1$ ), and had no influence in 126 cases. There were no cases in which SE had a downwards influence on BIRADS ordinality.

Table II. Ultrasonographic and clinical descriptive parameters of invasive lobular carcinoma tumors.

\begin{tabular}{ll}
\hline & $\begin{array}{l}\text { Number of tumors (\%) } \\
\mathbf{n}=\mathbf{1 8 0}\end{array}$ \\
\hline BI-RADS classification with SE & \\
$3-4 \mathrm{~A}$ & $2(1.11)$ \\
$4 \mathrm{~A}-4 \mathrm{~B}$ & $3(1.66)$ \\
$4 \mathrm{~B}-4 \mathrm{C}$ & $24(13.33)$ \\
$4 \mathrm{C}-5$ & $26(14.44)$ \\
B-mode US mass & $169(93.9)$ \\
Focality & \\
Uni-focal & $135(75)$ \\
Multi-focal & $33(18.33)$ \\
Multi-centric & $12(6.66)$ \\
Occult clinical assessment & $61(33.88)$ \\
Integumentary invasion & $17(9.44)$ \\
Nipple invasion & $1(0.55)$ \\
Edema & $5(2.77)$ \\
\hline
\end{tabular}

The data are expressed as number (\%); $\mathrm{n}$ - number of malignant lesions.
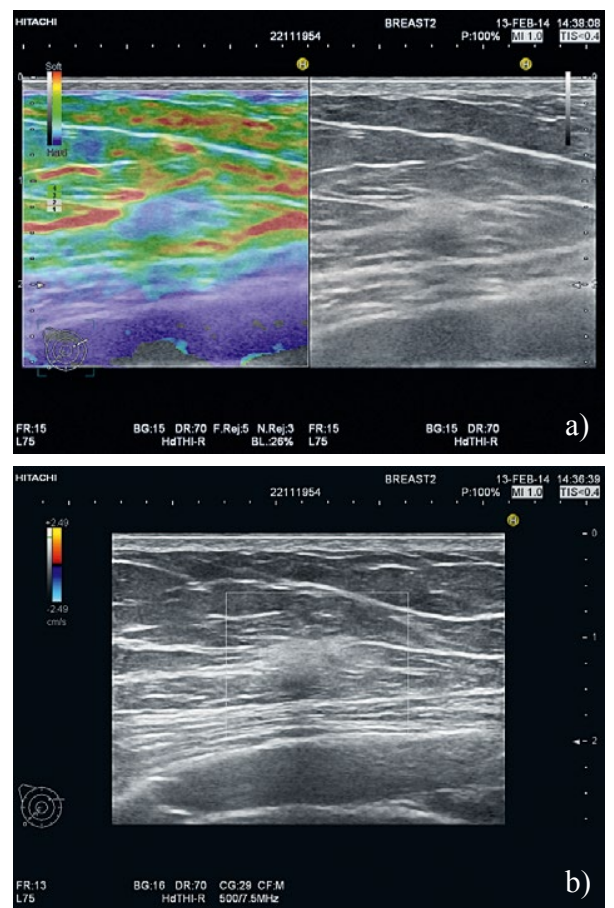

Fig 1. Doppler ultrasonography (a) and strain elastography (b) reveal a small hyperechoic lesion with indistinct margins, no vascular signal and rigid aspect on SE located in the in the right upper inner quadrant. The lesion was upgraded from BI-RADS 3 to BI-RADS 4A after the elastographic criteria was added. 


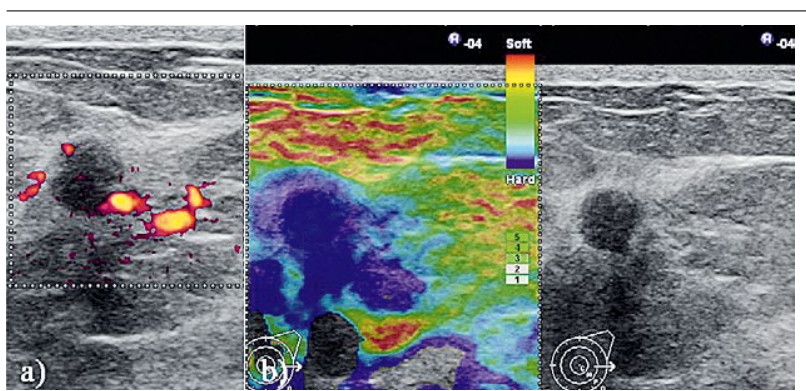

Fig 3. Doppler US reveals a hypoechoic round lesion with partially angulated margins and marginal vascularity (a). On strain elastography (b), the entire lesion and its surrounding parenchyma were shaded blue (Tsukuba score 5). The lesion was upgraded from BI-RADS $4 \mathrm{~B}$ to BI-RADS $4 \mathrm{C}$ after the elastographic criteria was added.

An ROC curve was used to determine a cut-off point for lesion size in relationship with BI-RADS change after $\mathrm{SE}(\mathrm{p}<0.000 ; \mathrm{AUC}=0.886)$. The largest Youden index was observed at $13 \mathrm{~mm}$ ILC size $(\mathrm{J}=0.670)$.

Finally, a binary logistic regression was performed to assess the relationship between tumor size and difference in BI-RADS classification. Results were statistically significant $\left(\chi^{2}=123.017, \mathrm{p}<0.000\right)$. The model explained between $49.5 \%-70.5 \%$ (Nagelkerke $R^{2}$ ) of the variance in the dependent variable and correctly classified $90.6 \%$ of cases. Small lesion $(<13 \mathrm{~mm})$ sizes were $17.92 \%$ more likely to influence BI-RADS classification (Fig. 3). We noted a smaller impact of tumors present in clinical assessment on BI-RADS classification $(\operatorname{Exp}(B)=0.64$, $\mathrm{p}<0.001$ ). Remaining independent variables (age, breast type, focality, clinical assessment, HCA, B-mode, and Doppler US examination) did not yield statistically significant values in the regression model.

\section{Discussions}

There is growing evidence pertaining to the importance of breast elastography in the BI-RADS classification [31]. SE and SWE in conjunction with BI-RADS are proven to increase diagnostic specificity and sensitivity [32]. Additionally, a relationship has been found between the elasticity imaging/B-mode ratio on SE and tumor grades in breast cancer [22]. A recent preliminary study has indicated that Contrast Enhanced Ultrasound (CEUS) provides superior diagnostic performance in sub-centimetre breast lesions, offering promising alternatives to biopsy [33]. Given these updates in the field of sonoelastography, we set out to explore the relationship between lesion size and the influence of SE on ILC classification, in order to identify an optimal size threshold at which SE adds significantly more value to the examination.

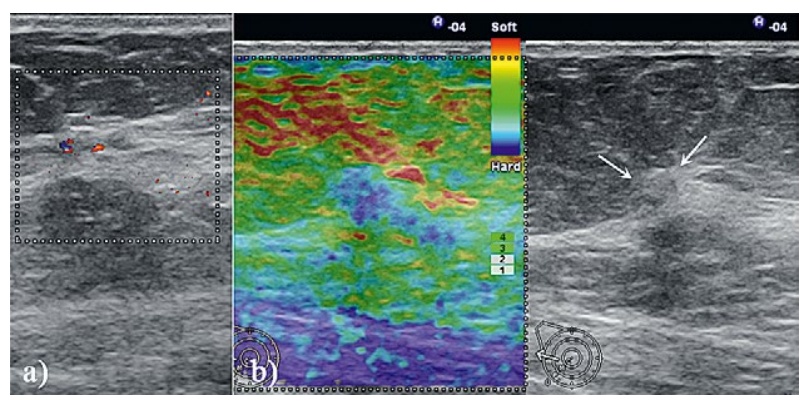

Fig 2. Doppler US indicated a 3-mm subtle lesion with hypoechoic appearance, indistinct margins (white arrows in b) and minimal marginal Doppler signal, classified BI-RADS 4A. The lesion appeared hard on SE (b). The classification was adjusted to BI-RADS 4B.

Due to diffuse growth patterns and the absence of microcalcifications, ILC is often times mammographically occult (between 21-43\%) [12,14,34]. Here lies the importance of breast US as an adjunct in breast cancer screening. Sim et al describes 4 cases of ILC that were normal at both mammography and greyscale US, but suspicious on SWE [21].

Similarly, our study builds current knowledge regarding elastographic imaging by demonstrating the added value of SE in small size lesions. Our study establishes that at $13 \mathrm{~mm}$ threshold in tumor size, SE is significantly more likely to influence BI-RADS classification. The binary logistic regression analysis brings high confidence evidence that our sample is not a random occurrence and is adjusted to relevant co-variates.

Another factor which this analysis controlled was the quality of B-mode visualization in small lesions. If the Bmode had been poorly visualized in small lesions, the impact of SE would have falsely accounted for our findings. We strongly reject this hypothesis because SE only marginally increased lesion classification, with most cases $(68.88 \%)$ with all cases being upgraded only to the next BI-RADS classification. If poor quality of small lesion B-mode was an issue, we would have observed higher discrepancies between BI-RADS scores that cannot be explained from a clinical perspective.

The potential to target the enhancement of BI-RADS classification via SE to small ILC is extremely important in clinical practice because it adds efficiency to the real-time sonographic examination. In a 955-patient study of Fleury et al [31] the addition of elastography to BI-RADS was proved to significantly increase diagnostic accuracy $(76.64 \%$ to $91.39 \%$ ) and specificity (from $72.07 \%$ to $80.65 \%$ ) of US in the assessment of breast lesions. As shown in our study, elastograms obtained aided the senologist in differentiating $3-4 \mathrm{~A}, 4 \mathrm{~A}-4 \mathrm{~B}, 4 \mathrm{~B}-4 \mathrm{C}$, 
or 4C-5 BI-RADS categories, but mostly in $<13 \mathrm{~mm}$ lesions. Therefore, the use of elastography in such lesions may have a critical role in patient management, including the decision whether to biopsy a lesion or not [35], according to BI-RADS guidelines.

A higher grade ILC can appear concealed on breast US due to microlobulated margins and acoustic enhancement. In this case, a higher Tsukuba score may raise question marks. Especially in small tumors with imprecisely delineated margins, this may trigger an important re-evaluation of the breast US and conjunct examinations in order to identify further malignancies or axillary lymphadenopathy. In this scenario, the screening process is significantly improved, positively impacting the treatment options and clinical management of the disease. Finally, an increased level of suspicion from the radiologist may provide additional information of value to the pathologist, as the differentiation between different tumor grades can be challenging and can vary among pathologists [22].

We must acknowledge that our research design is limited in its retrospective and cross-sectional approach. The study sample is conveniently drawn from a single outpatient clinic. Low breast cancer screening rates in Romania, along with differences in access to healthcare may skew the socioeconomic profile of study participants [36]. Moreover, the implication of a single observer is an additional source of potential bias. However, strict data collection methodology was enforced in order to limit reporting errors. Finally, the current study design may be improved (longitudinal/cohort) in order to increase inferential power. Future research opportunities may focus on integrating recent findings related to SE and SWE in a standardized protocol that provides a much needed consensus on the ideal breast US approach. An interesting additional avenue of research would be exploring the relationship between SE and Magnetic Resonance Elastography (MRE).

In conclusion, we report that SE impacts BI-RADS classification in ILC lesions smaller than $13 \mathrm{~mm}$. The need to perform SE as a standard component of breast cancer screening has cost implications. Our results require stronger sampling methods and mediation analysis in order to confirm study findings.

\section{Acknowledgements}

We acknowledge the assistance of Dr. Dan Tudor Eniu, MD, PhD from the Department of Surgical Oncology at the "Iuliu Hatieganu" University of Medicine and Pharmacy in Cluj-Napoca and Dr. Rareș Buiga MD, PhD from Santomar OncoDiagnostic Laboratory for their im- portant contributions to the surgical and histopathological components of this study.

Conflict of interest: none

\section{References}

1. DeSantis CE, Ma J, Goding Sauer A, Newman LA, Jemal A. Breast cancer statistics, 2017, racial disparity in mortality by state. CA Cancer J Clin 2017;6:439-448.

2. WHO $\mid$ Breast cancer: prevention and control. World Health Organization. Available from: http://www.who.int/cancer/ detection/breastcancer/en/. Accessed: October 1st 2017.

3. Yip CH, Buccimazza I, Hartman M, Deo SV, Cheung PS. Improving Outcomes in Breast Cancer for Low and Middle Income Countries. World J Surg 2015;3:686-692.

4. Vrdoljak E, Wojtukiewicz MZ, Pienkowski T, et al. Cancer epidemiology in Central, South and Eastern European countries. Croat Med J 2011;4:478-487.

5. Nelson HD, Cantor A, Humphrey L, et al. Screening for Breast Cancer: A Systematic Review to Update the 2009 U.S. Preventive Services Task Force Recommendation. Evidence Synthesis No. 124. AHRQ Publication No. 1405201-EF-1. Rockville, MD: Agency for Healthcare Research and Quality; 2016. Accessed: October 1st 2017.

6. Nelson HD, Fu R, Cantor A, Pappas M, Daeges M, Humphrey L. Effectiveness of Breast Cancer Screening: Systematic Review and Meta-analysis to Update the 2009 U.S. Preventive Services Task Force Recommendation. Ann Intern Med 2016;164:244-255.

7. Obenauer S, Hermann KP, Grabbe E. Applications and literature review of the BI-RADS classification. Eur Radiol 2005; 15:1027-1036.

8. Orel SG, Kay N, Reynolds C, Sullivan DC. BI-RADS categorization as a predictor of malignancy. Radiology 1999;211:845-850.

9. Lacquement MA, Mitchell D, Hollingsworth AB. Positive predictive value of the Breast Imaging Reporting and Data System. J Am Coll Surg 1999;189:34-40.

10. Drukteinis JS, Mooney BP, Flowers CI, Gatenby RA. Beyond mammography: new frontiers in breast cancer screening. Am J Med 2013;126:472-479.

11. Health Quality Ontario. Ultrasound as an Adjunct to Mammography for Breast Cancer Screening: A Health Technology Assessment. Ont Health Technol Assess Ser 2016;16:171.

12. Johnson K, Sarma D, Hwang ES. Lobular breast cancer series: imaging. Breast Cancer Res 2015;17:94.

13. Butler RS, Venta LA, Wiley EL, Ellis RL, Dempsey PJ, Rubin E. Sonographic evaluation of infiltrating lobular carcinoma. AJR Am J Roentgenol 1999;172:325-330.

14. Selinko VL, Middleton LP, Dempsey PJ. Role of sonography in diagnosing and staging invasive lobular carcinoma. J Clin Ultrasound 2004;32:323-332.

15. Sarvazyan A, Hall TJ, Urban MW, Fatemi M, Aglyamov SR, Garra BS. An overview of Elastography - An emerg- 
ing branch of medical imaging. Curr Med Imaging Rev 2011;7:255-282.

16. Zhi H, Ou B, Luo BM, Feng X, Wen YL, Yang HY. Comparison of ultrasound elastography, mammography, and sonography in the diagnosis of solid breast lesions. J Ultrasound Med 2007;26:807-815.

17. Itoh A, Ueno E, Tohno E, et al. Breast disease: clinical application of us elastography for diagnosis. Radiology 2006;239:341-350.

18. Barr RG, Destounis S, Lackey LB 2nd, Svensson WE, Balleyguier C, Smith C. Evaluation of breast lesions using sonographic elasticity imaging: a multicenter trial. J Ultrasound Med 2012;31:281-287.

19. Shi XQ, Li JL, Wan WB, Huang Y. A set of shear wave elastography quantitative parameters combined with ultrasound BI-RADS to assess benign and malignant breast lesions. Ultrasound Med Biol 2015;41:960-966.

20. Liu B, Zheng Y, Huang G, et al. Breast Lesions: Quantitative Diagnosis Using Ultrasound Shear Wave Elastography-A Systematic Review and Meta--Analysis. Ultrasound Med Biol 2016;42:835-847.

21. Sim YT, Vinnicombe S, Whelehan P, Thomson K, Evans A. Value of shear-wave elastography in the diagnosis of symptomatic invasive lobular breast cancer. Clin Radiol 2015;70:604-609.

22. Grajo JR, Barr RG. Strain Elastography for Prediction of Breast Cancer Tumor Grades. J Ultrasound Med 2014;33:129-134.

23. Hao SY, Ou B, Li LJ, et al. Could ultrasonic elastography help the diagnosis of breast cancer with the usage of sonographic BI-RADS classification? Eur J Radiol 2015;84:2492-2500.

24. Moukhtar FZ, ElMaati AAA. Real-time tissue elastography combined with BIRADS-US classification system for improving breast lesion evaluation. Egypt J Radiol Nucl Med 2014;45:1021-1028.

25. Chiorean A, Duma MM, Dudea S, et al. Real-time ultrasound elastography of the breast: state of the art. Med U1trason 2008;10:73-82.
26. Lee SH, Chang JM, Cho N, et al. Practice guideline for the performance of breast ultrasound elastography. Ultrasonography 2014;33:3-10.

27. Mainiero MB, Lourenco A, Mahoney MC, et al. ACR Appropriateness Criteria - Breast Cancer Screening. J Am Coll Radiol 2016;13:R45-R49.

28. Barr RG. Sonographic breast elastography: a primer. J U1trasound Med 2012;31:773-783.

29. Barr RG, Nakashima K, Amy D, et al. WFUMB Guidelines and Recommendations for Clinical Use of Ultrasound Elastography: Part 2: Breast. Ultrasound Med Biol 2015;41:1148-1160.

30. Lynch SP, Lei X, Chavez-MacGregor M, et al. Multifocality and multicentricity in breast cancer and survival outcomes. Ann Oncol 2012;23:3063-3069.

31. Fleury Ede F. The importance of breast elastography added to the BI-RADS $\AA$ (5th edition) lexicon classification. Rev Assoc Med Bras (1992) 2015;61:313-316.

32. Wang M, Yang Z, Liu C, et al. Differential Diagnosis of Breast Category 3 and 4 Nodules Through BI-RADS Classification in Conjunction with Shear Wave Elastography. Ultrasound Med Biol 2017;43:601-606.

33. Xiao X, Jiang Q, Wu H, Guan X, Qin W, Luo B. Diagnosis of sub-centimetre breast lesions: combining BIRADS-US with strain elastography and contrast-enhanced ultrasound-a preliminary study in China. Eur Radiol 2017;27:2443-2450.

34. Brem RF, Ioffe M, Rapelyea JA, et al. Invasive lobular carcinoma: detection with mammography, sonography, MRI, and breast-specific gamma imaging. AJR Am J Roentgenol 2009;19:379-383.

35. Bojanic K, Katavic N, Smolic M, et al. Implementation of Elastography Score and Strain Ratio in Combination with B-Mode Ultrasound Avoids Unnecessary Biopsies of Breast Lesions. Ultrasound Med Biol 2017;43:804-816.

36. EUROSTAT Statistic Explained. EHIS WAVE I. Breast cancer screening statistics. Available from: http://ec.europa. eu/eurostat/statistics-explained/index.php/Breast_cancer_ screening_statistics; Accessed: October 1st 2017. 